\title{
RADIOGRAPHIC EVALUATION OF CRYSTAL BONE LEVEL OF IMMEDIATE LOADED IMPLANT RESTORED WITH TWO DIFFERENT CAD-CAM MATERIALS
}

\author{
Noura Turky Saad* , Ehab A Farghaly**, Mohamed Shokry**, \\ Ahmed M Abdelhamid ${ }^{* * * *}$ and Essam Osman ${ }^{* * * * *}$
}

\begin{abstract}
Purpose: This study was conducted to radiographically evaluate the effect of two different superstructure CAD/CAM fabricated materials, on crestal bone loss around immediately loaded implant.
\end{abstract}

Materials and Methods: A total of twelve healthy patients having mandibular bilateral missing first molars with acceptable bone volume were selected based on specific inclusion and exclusion criteria, each patient received a CAD/CAM monolithic zirconia, in one site, while the other site received a CAD/CAM Enamic superstructure, in 48 hours after implant insertion. Implants and abutments were examined for stability, gingival and periodontal health prior to crown cementation. Radiographic evaluation was done immediately at the time of crown cementation then at 6 and 12 months.

Results: Marginal bone loss was measured at two points, the most buccal crestal bone, and the most lingual crestal bone. Radiographic results showed no significant difference regarding mean MBL between Zirconia and Enamic on 6 and 12 months.

Conclusion: Immediate loading of a single mandibular molar implant with the final restoration seem to be a reliable technique when certain parameters are respected. Type of implant superstructure material has no significant influence on the bone supporting the implant within the time period of this study.

* BDS, Msc of Prosthodontics, Beirut Arab University. Lebanon

** Assis. Professor of Prosthodontics, Beirut Arab University, Faculty of Dentistry, Lebanon.

*** Assis. Professor of Oral and Maxillofacial Surgery, Beirut Arab University, Faculty of Dentistry, Lebanon, and Alexandria University, Egypt

**** Professor of Prosthodontics Faculty of Dentistry, Alexandria University, Egypt.

****** Professor of Dental Biomaterials, Faculty of Dentistry, Beirut Arab University, Lebanon. 


\section{INTRODUCTION}

Replacement of missing teeth has become one of the most important needs for patients attending clinics seeking esthetics and/or function. Many treatment modalities are available for replacing a single missing tooth; removable partial denture, fixed partial denture or dental implant. Each modality is a possible treatment option and has its own benefits and drawbacks ${ }^{1}$.

Alveolar bone is better preserved when restoring extracted teeth by dental implant, as dental implants integrate with jawbone, helping to keep the bone healthy and intact ${ }^{2}$.

However, dental implants differ from the natural teeth in the biomechanical behavior and its relation to bone. In natural teeth, the periodontal membrane acts as a cushion dissipating forces, protecting bone from the harmful effect of biting forces. While in case of implants, all forces are transmitted directly to bone specifically around the coronal portion of the implant that may lead to crestal bone loss ${ }^{3}$.

The longevity of dental implants depend on a number of complex interlocking factors, on top of them is the biomechanical factors and due to intimate contact at the bone-implant interface, implant durability is directly linked to the selection of appropriate implant position, prosthesis design, biocompatibility as well as mechanical and physical properties of the materials of the implant superstructure $^{4}$.

Immediate implants are placed with adequate primary stability; its corresponding restoration with full centric occlusion in maximum intercuspation must be placed within 48 hours post-surgery. Success of this process is based on design and material of the restoration to prevent micro motion of the implant ${ }^{5}$.

Milled ceramics are most relevant to restorative dentists and where the greatest changes in clinical practice have been realized. A majority of crownand-bridge restorations are now produced through
CAD/CAM, often with new ceramic materials.CAD/ CAM ceramic materials evolved from traditional feldspathic porcelain, an esthetic but low-strength, brittle material, to a range of materials with different strength, resilience, and esthetic properties. They are clinically successful and are replacing porcelainfused-to-metal (PFM) restorations ${ }^{6}$.

\section{MATERIALS AND METHODS}

A total of 14 patients in the age range $20-50$ years having bilateral first molar missing site with the presence of adjacent and opposing teeth intact with a good periodontal and general systemic health were selected from the outpatient clinic at the Faculty of Dentistry, Beirut Arab University. Each patient was given a detailed verbal and written description of the risks and benefits of the proposed treatment. They were required to sign a consent form prior to the procedure and presented to the IRB committee of Beirut Arab University for approval.

An aseptic surgical technique was followed. Antimicrobial prophylaxis included amoxicillin 500 $\mathrm{mg}$ three times daily for 5 days, starting $1 \mathrm{~h}$ before surgery and post-surgery analgesic treatment was ibuprofen $400 \mathrm{mg}$ twice daily for 3 days. Implants were placed by one operator using a 3D-printed acrylic resin surgical guide template processed through a 3D printing (Formlabs 3-Shape USA) of the digital impression of each implant site. Patients were treated under local anesthesia, surgical site was examined to fit in required bone volume and similar implant length and diameter $(4.1 \times 10 \mathrm{~mm})$ was selected for all cases. After punch on a healed site, an initial pilot drill was passed through the surgical guide to the depth corresponding to the length of the implant chosen. Next intermediate drills of the diameter and length of the implant were used to expand the osteotomy. The implant with its attached delivery post was placed into the prepared site with gentle digital pressure until resistance is met and seated into final position using torque ratchet to 
$30 N$. Reading of the implant stability was recorded with the help of a resonance frequency analyzer osstell - ISQ.

Open try impression technique was used for cast fabrication using type IV extra hard stone, impression coping replaced by abutment then scanned using a CAD-CAM scanner (Sirona InEos $\mathrm{X} 5$ ). The opposing diagnostic cast and bite were also scanned; occlusal clearness was checked on virtual articulator.

With the aid of Sirona inLab SW CAD 15 software abutments margins were traced and crowns were designed to produce Stereolithography STL file which sent to the milling machine (Sirona inLab CM X5); for Zirconia, crowns were milled then cleaned in ultrasonic solution, dried and sintered according to manufacturer directions. For Enamic crowns, finishing and polishing was completed using manufacturer-supplied kit. All patients were restored one side with zirconia, and other side with enamic crowns and cemented with u-200 (3M) resin cement on its straight corresponding abutment. Cementation was done extraorally, then crown-abutment unite was screwed to the implant intraorally after 48 hours of implant placement. Bone level evaluation was done using radiographic parameters and statistical data was analyzed.

\section{Radiographic parameters assessed}

The level of alveolar bone around each implant was evaluated using cone beam computed tomography $(\mathrm{CBCT})^{7}$. For each patient, three CBCT images were captured (at time of loading, three, and six-months) on lower jaw, to evaluate the changes in crestal bone level with respect to the implant.

In curved slicing, the coronal plane represented by a line drawn in a path passing through the center of implant placed in bone, to form the dental arch, thus, making it almost drawn in the same position in each repeated CBCT.

In order to take measurements exactly in the same position in each following CBCT, a fixed reference line was marked tangent to apical tip of implant, then a line drawn from crest of bone buccal and lingual perpendicular to the horizontal tangent. This line will represent the level of the bone in the sagittal plane (Figure ).

Readings at intervals 0,6 , and 12 months, reflecting the changes in crestal bone level around implant which was measured at the highest level of bone on buccal and lingual side of each crown. Marginal bone loss was measured at two points; buccal and lingual crestal bone.

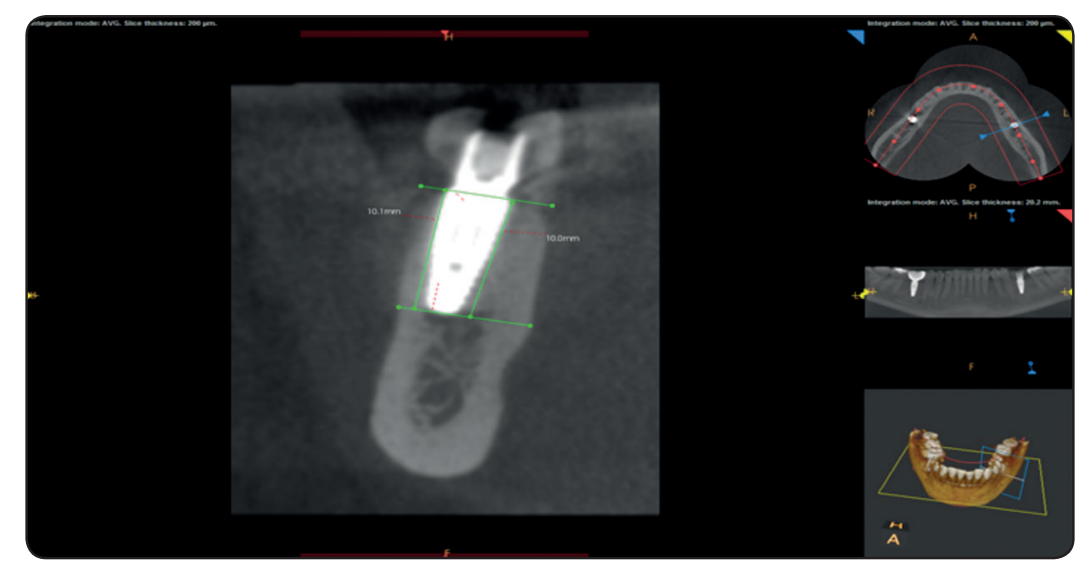

Figure: CT image showing the line drawn from crest of bone buccal and lingual perpendicular to the horizontal tangent. 


\section{RESULTS}

\section{Marginal Bone Loss (MBL):}

Comparison of MBL between zirconia and enamic crowns at both sites was not significant. When measuring bone loss at the most buccal and most lingual crestal bone, the study showed no significant difference regarding mean MBL between zirconia and enamic in 6 and 12 months.

\section{Effect of time periods on MBL in each site:}

In implants restored with zirconia crowns, both buccal and lingual bone loss showed a non-significant difference. In implants restored with enamic crowns, both buccal and palatal showed no significant decrease in MBL. Data presented in the Table.

TABLE: Bone height calibration in $\mathrm{mm}$ at different time in buccal and lingual side in the two studied groups (Zirconia and Enamic).

\begin{tabular}{|c|c|c|c|}
\hline & Zirconia & Enamic & $P$ value \\
\hline \multicolumn{4}{|l|}{ I. Buccal } \\
\hline \multicolumn{4}{|l|}{ Baseline: } \\
\hline Range & $8.7-10.7$ & $8.6-10.7$ & \\
\hline Mean \pm S.D. & $10.0 \pm 0.62$ & $9.9 \pm 0.71$ & 0.321 N.S. \\
\hline \multicolumn{4}{|l|}{6 months: } \\
\hline Range & $8.6-10.5$ & $8.7-10.8$ & \\
\hline Mean \pm S.D. & $9.8 \pm 0.76$ & $10.0 \pm 0.70$ & 0.411 N.S. \\
\hline \multicolumn{4}{|l|}{12 months: } \\
\hline Range & $8.6-10.5$ & 8.7-10.8 & \\
\hline Mean \pm S.D. & $9.8 \pm 0.73$ & $10.0 \pm 0.74$ & 0.354 N.S. \\
\hline \multicolumn{4}{|l|}{ II. Lingual } \\
\hline \multicolumn{4}{|l|}{ Baseline: } \\
\hline Range & $8.5-11.2$ & 8.4-10.8 & \\
\hline Mean \pm S.D. & $10.21 \pm 0.82$ & $10.2 \pm 0.83$ & 0.958 N.S. \\
\hline \multicolumn{4}{|l|}{6 months: } \\
\hline Range & $8.3-11.0$ & $8.5-11.1$ & \\
\hline Mean \pm S.D. & $10.1 \pm 0.91$ & $10.2 \pm 0.82$ & 0.892 N.S. \\
\hline \multicolumn{4}{|l|}{12 months: } \\
\hline Range & $8.3-11.0$ & $8.5-11.1$ & \\
\hline Mean \pm S.D. & $10.1 \pm 0.93$ & $10.2 \pm 0.85$ & 0.901 N.S. \\
\hline
\end{tabular}

(N.S.: not significant at level 0.05)

\section{DISCUSSION}

In this study, a comparative evaluation of immediate implant restored with enamic versus zirconia restoration. The treatment protocol involved fabrication of crowns within 48 hours. During surgery, a guided method used, punch and drilling through it to reach final drill and implant insertion, with less resultant discomfort and swelling because muscle attachment is uninvolved and smaller punch with less resultant bone loss ${ }^{8}$.

The edentulous area was bounded by neighboring teeth in a normal contact condition with no rotation since the proximity of the proximal contour of neighboring teeth is an essential factor to ensure adequate bone height, and complete fullness of the papilla in the interdental embrasure space ${ }^{9}$.

Implants was placed entirely within bone and away from significant anatomic structures (e.g., the inferior alveolar canal). $10 \mathrm{~mm}$ of vertical bone dimension and $8 \mathrm{~mm}$ of horizontal was available for implant placement. These dimensions prevented encroachment on anatomic structures and allow a minimum of $2.0 \mathrm{~mm}$ of bone on both the lingual and facial aspect of the implant ${ }^{10}$.

CBCT has been regarded as a high-quality reliable image-acquisition method for the dental maxillofacial area, in comparison with other tomographic methods ${ }^{11}$. Surgical guide was manufactured with the aid of CBCT and CAD-CAM to estimate the orientation and location of the implant to be inserted before implant placement ${ }^{12}$, to insure all the implants are placed precisely in their accurate positions ${ }^{13}$, with less chances of error, and offers reproducibility and hence minimizes the presence of variables during implant placement ${ }^{14}$.

Drilling for implant site was done by using the manufacture drills starting with the pilot drill, to insure proper parallelism of the implant positioning. The wrench turned the screw until a recommended torque of $35 \mathrm{Ncm}$ was applied. Final drilling was 
done cancel to get full contact between the threads of the implant and the surrounding bone which will have the great effect on primary stability, and load transfer from the implant to the supporting tissues ${ }^{15}$.

Zirconia and enamic was selected in this study as crown materials because of their good esthetics coupled with biocompatibility, it was seen that the zirconia crowns exhibited the least marginal gingival inflammation ${ }^{16}$, taking into consideration that glazed surfaces show less plaque accumulation than non-glazed surfaces ${ }^{17}$.

Monolithic zirconia and Enamic crowns was fabricated with no fear from causing more wear to the opposing dentition, since according to EsquivelUpshaw in $2018^{18}$, monolithic zirconia exhibited non significant wear of opposing enamel compared to metal-ceramic crowns and control enamel after one year.

Restorations were designed and milled using CAD/CAM technology to insure exact monolithic copies of final crowns, producing a perfectly contoured interim restoration, since gingival recession and inflammation is directly related to marginal adaptation, axial contours ${ }^{19}$, in addition to all advantages of such technology including rapid production, improved wear properties, decreased laboratory fees and improved cross infection control ${ }^{20}$.

Cone beam computed tomography (CBCT) was chosen as it provides detailed anatomy of teeth, bone, and the changes in the alveolar area around implant in multiple planes. Data can be oriented so that patient's anatomic features are realigned which helps in standardization of images. In addition to cursor-driven images that provide the clinician with dimensional assessment that are free from distortion and magnification ${ }^{21}$. Therefore, measurement will be taken exactly in the same position in each x-ray cut every time.

Some studies, suggested that radiographic interpretation of the alveolar bone levels has proved to be one of the most valuable tools for evaluation of implant success ${ }^{11,22}$. Variable results regarding bone levels of implants with time was stated by some researchers and can be found in the data of several other studies ${ }^{5,23}$. In addition, effect regarding crown material with peak masticatory stresses of osseointegrated implant prosthesis was studied by Hobkirk, J. A., \& Psarros, $\mathrm{K}^{24}$, and, Yuan J.C., \& Sukotjo C. ${ }^{25}$ whom stated that no difference was found regarding load rates with using ceramic or resin prostheses and this with accordance with our results. Results of the present study showed that the amount of bone loss with immediate loading implants showed higher values with zirconia crown than with enamic crown. Bone loss in zirconia site of immediate loading group was $0.3 \mathrm{~mm}$ while in the enamic site it was $0.2 \mathrm{~mm}$. These values agreed with Boronat, et al ${ }^{26}$. Immediate loading protocols create excessive load that exceed the loading capacity of the interfacial bone, furthermore, slight load on healing bone shortens healing rather than prolong it and the bone tissues adapt their trabeculae to the accepted magnitude and direction of the load. Also Galal, et al. ${ }^{23}$, found that immediate loading showed more bone loss than conventional delayed loading protocol. On the contrary, Kushaldeep, et al. ${ }^{27}$, found that there is no difference between the immediate and delayed loading protocols.

Comparison between bone-loss with the two superstructures crown materials revealed nonstatistically significant difference between zirconia and enamic restorations through the whole study period. These results were in agreement with Sertgoz ${ }^{28}$, who stated that using a prosthetic superstructure with lower elastic modulus neither led to substantial difference in stresses over cortical and spongy bone around implants. Hardness of a material was related to the stress absorption from impact loads. 


\section{SUMMARY}

This study was conducted to radiographically evaluate and compare a relatively CAD/CAM hybrid material (Enamic) used as crown for immediately loaded single implant with a CAD/CAM monolithic Zirconia, using CBCT to measure crestal bone loss, it was found out that no significant difference regarding mean MBL between zirconia and enamic in 6 and 12 months.

\section{REFERENCES}

1. Christensen G. J. (2000). Elective vs. Mandatory Dentistry. Journal of American Dental Association, 131(10):149-68.

2. Esposito, M., \& Worthington, H. V. (2013). Interventions for Replacing Missing Teeth: Hyperbaric Oxygen Therapy for Irradiated Patients Who Require Dental Implants. Cochrane Database of Systematic Reviews, (9).

3. Tsouknidas, A., Giannopoulos, D., Savvakis, S., Michailidis, N., Lympoudi, E., Fytanidis, D. \& Michalakis, K. (2016). The Influence of Bone Quality on the Biomechanical Behavior of a Tooth-Implant Fixed Partial Denture: A Three-Dimensional Finite Element Analysis. International Journal of Oral \& Maxillofacial Implants, 31(6).

4. Haiat, G., Wang, H. L., \& Brunski, J. (2014). Effects of Biomechanical Properties of the Bone-Implant Interface on Dental Implant Stability: From in Silico Approaches to the Patient's Mouth. Annual Review of Biomedical Engineering, 16: 187-213.

5. Glauser, R., Ree, A., Lundgren, A., Gottlow, J., Hammerle, C. H., \& Scharer, P. (2001). Immediate Occlusal Loading of Brånemark Implants Applied in Various Jawbone Regions: A Prospective, 1-Year Clinical Study. Journal of Clinical Implant Dentistry and Related Research, 3(4): 204-213.

6. Della Bona A, Kelly JR. The clinical success of all ceramic restorations. J Am Dent Assoc.2008;139 Suppl:8S-13S.

7. Mahrous AI, Aldawash HA, Soliman TA, Banasr FH, Abdelwahed A. Implant supported distal extension over denture retained by two types of attachments. A comparative radiographic study by cone beam computed tomography. $\mathrm{J}$ Int Oral Health 2015;7(5):5-10.

8. Wadhwa B, Jain V, Bhutia O, Bhalla AS, Pruthi G. Flapless versus open flap techniques of implant placement: A 15-month follow-up study. Indian J Dent Res 2015; 26:372-7.
9. Gracis, S., Fradeani, M., Celletti, R., \& Bracchetti, G. (2001). Biological integration of aesthetic restorations: factors influencing appearance and long-term success. Journal of Periodontology 2001, 27(1), 29-44..

10. Misch. C. E., Steigenga, J., Barboza, E., Misch-Dietsh, F., Cianciola, L. J. \& Kazor, C. (2006). Short Dental Implants in Posterior Partial Edentulism: A Multicenter Retrospective 6-Year Case Series Study. Journal of Periodontology, 77: 1340-1347.

11. Reinhilde Jacobs, Benjamin Salmon, Marina Codari, Bassam Hassan, and Michael M. Bornstein. Cone beam computed tomography in implant dentistry: recommendations for clinical use. BMC Oral Health Journal. 2018; 18: 88..

12. Kassebaum, D. K., Nummikoski, P. V., Triplett, R. G., \& Langlais, R. P. (1990). Cross-Sectional Radiography for Implant Site Assessment. Journal of Oral Surgery, Oral Medicine, Oral Pathology, 70(5): 674-678.

13. Kola, M. Z., Shah, A. H., Khalil, H. S., Rabah, A. M., Harby, N. M. H., Sabra, S. A., \& Raghav, D. (2015). Surgical Templates for Dental Implant Positioning; Current Knowledge and Clinical Perspectives. Nigerian Journal of Surgery, 21(1): 1-5.

14. D'haese, J., Ackhurst, J., Wismeijer, D., De Bruyn, H., \& Tahmaseb, A. (2017). Current State of the Art of Computer-Guided Implant Surgery. Journal of Periodontology, 73(1): 121-133.

15. Rahimi, A., Bourauel, C., Jager, A., Gedrange, T., \& Heinemann, F. (2009). Load Transfer by Fine Threading the Implant Neck-A FEM Study. Journal of Physiology \& Pharmacology, 60(Suppl 8): 107-112.

16. Saravanakumar, P. (2017). Effect of Different Crown Materials on the InterLeukin-One Beta Content of Gingival Crevicular Fluid in Endodontically Treated Molars: An Original Research. Cureus, 9(6):e 1361.

17. Satheesh B. Haralur. Evaluation of efficiency of manual polishing over autoglazed and overglazed porcelain and its effect on plaque accumulation. Journal of advanced prosthodontics, 4(4), 179-186.

18. Esquivel-Upshaw, J. F., Kim, M. J., Hsu, S. M., Abdulhameed, N., Jenkins, R., Neal, D., ... \& Clark, A. E. (2018). Randomized clinical study of wear of enamel antagonists against polished monolithic zirconia crowns. Journal of Dentistry, 68, 19-27.

19. Burns, J. W., Glenn, B., Bruehl, S., Harden, R. N., \& Lofland, K. (2003). Cognitive factors influence outcome 
following multidisciplinary chronic pain treatment: a replication and extension of a cross-lagged panel analysis. Behaviour research and therapy, 41(10), 1163-1182.

20. Freedman M, Quinn F, O’Sullivan M. Single unit CAD/ CAM restorations: a literature review. J Ir Dent Assoc. 2007;53(1):38-45. .

21. 21- Scarfe WC, Farman AG. What is cone-beam CT and how does it work? Dent Clin North Am. 2008;52(4):707-v.

22. Lewin S, Riben C, Thor A, Öhman-Mägi C. Bone Volume Assessment Around Dental Implants After Open Maxillary Sinus Elevation Surgery: A Quantitative Approach to CBCT Images. Int J Oral Maxillofac Implants. 2019 March/April;34(2):489-498.

23. Galal, R. M., Yousief, S. A., \& Alqahtani, W. M. (2018). Clinical and Radiographic Outcomes of Three Different Loading Protocols for Single-Implant-Supported Prostheses: A Randomized Controlled Trial. Journal of International Oral Health, 10(4): 180-186.

24. Hobkirk, J. A., \& Psarros, K. (1992). The Influence of Occlusal Surface Material on Peak Masticatory Forces Us- ing Osseointegrated Implant-Supported Prostheses. International Journal of Oral \& Maxillofacial Implants, 7(3): 67-82.

25. Yuan JC, Sukotjo C. Occlusion for implant-supported fixed dental prostheses in partially edentulous patients: a literature review and current concepts. J Periodontal Implant Sci. 2013;43(2):51-57.

26. Boronat López A, Balaguer Martínez J, Lamas Pelayo J, Carrillo García C, Peñarrocha Diago M. Resonance frequency analysis of dental implant stability during the healing period. Med Oral Patol Oral Cir Bucal. 2008;13(4):E244-E247. .

27. Kushaldeep, A. T., Upadhyaya, V., \& Raghuvanshi, M. (2018). Comparative evaluation of the influence of immediate versus delayed loading protocols of dental implants: A radiographic and clinical study. The Journal of the Indian Prosthodontic Society, 18(2), 131.

28. Sertgöz, A. (1997). Finite Element Analysis Study of the Effect of Superstructure Material on Stress Distribution in an Implant-Supported Fixed Prosthesis. International Journal of Prosthodontics, 10(1):19-27. 